\title{
The Modulation of Presynaptic Inhibition in Single Muscle Primary Afferents during Fictive Locomotion in the Cat
}

\author{
Ariane Ménard, Hugues Leblond, and Jean-Pierre Gossard \\ Centre de Recherche en Sciences Neurologiques, Département de Physiologie, Faculté de Médecine, Université de \\ Montréal, Montréal, Québec, Canada, H3C 3J7
}

The aim of this study is to understand the functional organization of presynaptic inhibition in muscle primary afferents during locomotion. Primary afferent depolarization (PAD) associated with presynaptic inhibition was recorded intra-axonally in identified afferents from various hindlimb muscles in L6-L7 spinal segments during fictive locomotion in the decerebrate cat. PADs were evoked by the stimulation of peripheral muscle nerves and were averaged in the different epochs of the fictive step cycle. Fifty-three trials recorded from 39 muscle axons (37 from group I and two from group II) were retained for analysis. The results showed that there was a significant phasedependent modulation of PAD amplitude $(p<0.05)$ in a majority of muscle afferents (30 of $39,77 \%$ ). However, not all stimulated nerves led to significantly modulated PADs in a given axon (36 of 53 trials, $68 \%$ ). We also observed that the pattern of modulation (phase for maximum and minimum PAD amplitude and the depth of modulation) varied with each recorded afferent, as well as with each stimulated nerve. We further evaluated the effect of PAD modulation on the phasic transmission of the monosynaptic reflex (MSR) and found that PADs decreased the MSR amplitude in all phases of the fictive step cycle, independent of the PAD pattern in individual group I fibers. We conclude that (1) PAD modulation patterns of all group I fibers contacting motoneurons led to an overall reduction in monosynaptic transmission, and (2) individual PAD patterns could participate in the control of transmission in specific reflex pathways during locomotion.

Key words: primary afferent depolarization (PAD); presynaptic inhibition; muscle group I fibers; fictive locomotion; spinal cord; motor control
Although the central pattern generator (CPG) (Grillner, 1981) in mammalian spinal cord is able to produce complex locomotor patterns in the absence of sensory input, sensory feedback in general, and proprioceptive input in particular, is needed for adaptive modifications of the step cycle (Rossignol, 1996). For instance, proprioceptive input from muscles is used to adjust the timing and amplitude of muscle contractions during the different phases of the step cycle (Pearson and Duysens, 1976; Conway et al., 1987; Gossard et al., 1994a; McCrea et al., 1995; Hiebert et al., 1996). To be meaningful, the efficacy of proprioceptive feedback must be phasically controlled to induce adaptive responses in the proper phase of the locomotor cycle. Peripheral (e.g., gamma innervation of muscle spindles), as well as central, mechanisms participate in the phasic modulation of transmission in proprioceptive pathways.

One powerful central mechanism by which the CPG could control sensory feedback is presynaptic inhibition of primary afferent terminals. Indeed, there are GABAergic interneuronal networks in the spinal cord capable of decreasing transmitter release by evoking a primary afferent depolarization (PAD) through axoaxonic contacts (Eccles, 1961; Schmidt, 1971; Nicoll

Received Aug. 11, 1998; revised Sept. 29, 1998; accepted Oct. 14, 1998.

This work funded by a grant awarded to J.-P.G. from the Medical Research Council of Canada and from the Fonds pour la Formation de Chercheur et l'Aide á la Recherche (FCAR) du Québec. A.M. was supported by the Natural Sciences and Engineering Council of Canada (NSERC). H.L. was supported by the Rick Hansen Man in Motion Foundation. We thank Dr. T. Drew for reviewing this manuscript.

Correspondence should be addressed to Dr. Jean-Pierre Gossard, Centre de Recherche en Sciences Neurologiques, Département de Physiologie, Faculté de Médecine, Université de Montréal, C.P. 6128, Succ. Centre-ville, Montréal, Québec, Canada, H3C 3 J7.

Copyright (C) 1998 Society for Neuroscience $\quad 0270-6474 / 98 / 190391-09 \$ 05.00 / 0$ and Alger, 1979). Presynaptic inhibition has been proposed often to explain changes in the amplitude of the monosynaptic reflex (MSR) during walking in humans (Stein and Capaday, 1988; Yang and Whelan, 1993). Moreover, when PADs reach firing threshold, the evoked discharges are antidromically propagated in primary afferent axons and may collide with incoming volleys and even change the excitability of peripheral sensory receptors (Tonnies, 1939; Lindblom, 1958; Loewenstein, 1959, Ito, 1968; Bévengut et al., 1997). For example, rhythmic bursts of antidromic discharges can be found in a majority of cut dorsal rootlets of decerebrated cats walking on a treadmill (Beloozerova and Rossignol, 1994, 1995). The phasic timing of such discharges suggests that the underlying PADs are strongly influenced by the CPG. It is thus important to know more about the role of PAD pathways in the control of locomotion. There are, however, few studies focused on the transmission in PAD pathways during locomotion in mammals. Duenas et al. (1990) evoked antidromic discharges in dorsal rootlets belonging to the triceps surae muscles by stimulating cutaneous nerves during stepping in thalamic cats and found that maximal discharges occurred during the extensor phase of the step cycle. Similarly, previous intracellular studies of PADs in cutaneous afferents during fictive locomotion showed that the amplitude of the evoked PAD was maximal during the extensor phase (Gossard and Rossignol, 1990; Gossard et al., 1990). To our knowledge, there is yet no study investigating directly the changes in PAD amplitude in individual muscle afferents during locomotion. We thus used intra-axonal recordings to study PADs in single primary afferents from hindlimb muscles of the decerebrate cat during fictive locomotion. We also evaluated directly, and for the first time, the effect of PADs on the MSR amplitude during fictive locomotion. 

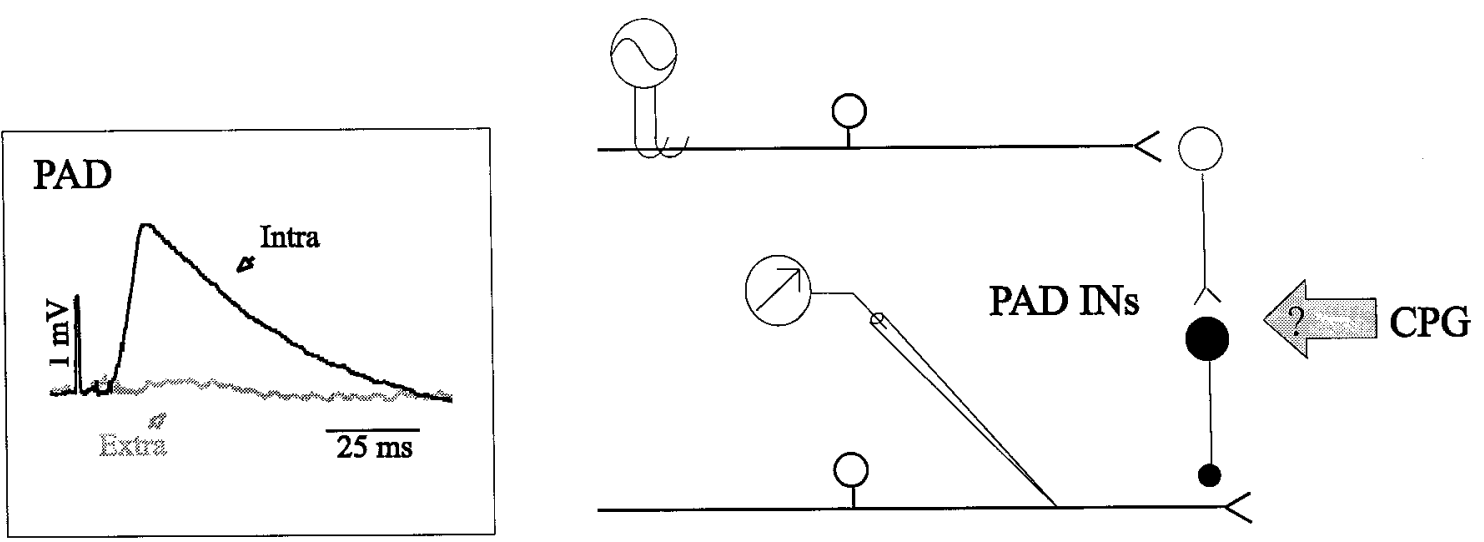

Figure 1. Experimental setup and recordings. Schematic representation of the spinal cord pathways under study. Right, A stimulated muscle afferent contacts a short chain of two interneurons (PAD INs) responsible for PAD. The PAD is mediated by an axoaxonic synapse located at the terminal of a muscle afferent recorded with a micropipette. This pathway is stimulated, whereas the CPG for locomotion is activated. Left, Averaged PAD recorded intra-axonally in a FDHL group I fiber (Intra, black trace) evoked by a stimulation of PBSt $(3 \mathrm{p}, 5 \mathrm{~T}, 300 \mathrm{~Hz})$ superimposed on the extra-cellular potential (Extra, gray trace) evoked by the same stimulation.

Some of these results have been reported previously in abstract form (Ménard et al., 1997).

\section{MATERIALS AND METHODS}

Preparation. The experiments were performed on 21 cats of either sex $(2.5-4.3 \mathrm{~kg})$. All animal experiments were conducted according to the Guide for the Care and Use of Experimental Animals (Canada), using protocols approved by the Ethics Committee of Université de Montréal. Cats were first anesthetized by inhalation of a mixture of halothane (usually $2.0-3.0 \%$ ), oxygen (50\%), and nitrous oxide (50\%) through a mask and then through an intratracheal tube for the rest of the surgery. Cannulas were inserted in the right common carotid artery for monitoring the blood pressure and were inserted in one jugular vein and one cephalic vein for administration of fluids. All animals received an intravenous injection of prednisolone sodium succinate $(1 \mathrm{mg} / \mathrm{kg}$; Solu-deltacortef; Upjohn, Ontario, Canada) to minimize the shock and brainstem swelling after decerebration, and when necessary, norepinephrine bitartrate (Levophed; Sanoti-Winthrop) was given to maintain blood pressure within physiological limits. A subcutaneous injection of atropine sulfate $(0.4 \mathrm{mg})$ to prevent excessive secretions was performed, as well as an intramuscular injection of furosemide $(5 \mathrm{mg} / \mathrm{kg}$; Lasix; Hoechst) when a diuretic effect was desired.

The following selected flexor and extensor muscle nerves from the left hindlimb were dissected free and mounted on bipolar silver chloride electrodes for recording [electroneurogram (ENG)] and stimulation purposes: posterior biceps-semitendinosus (PBSt), semimembranosusanterior biceps (SmAB), lateral gastrocnemius-soleus (LGS), medial gastrocnemius (MG), plantaris (Pl), flexor digitorum longus and flexor hallucis longus together (FDHL), the rest of tibialis posterior (Tib), tibialis anterior and extensor digitorum longus together (TA/EDL), and the sciatic nerve just below PBSt. Among these nerves, only PBSt and SmAB were cut.

After a laminectomy exposing spinal segments L4-L7, the cats were transferred to a stereotaxic frame, and an extensive craniotomy was performed. The cat was then decerebrated by making a precollicular postmammillary transection with a spatula and removing the rostral brain tissue by aspiration. Anesthesia was discontinued, and the cats were paralyzed with gallamine triethiodide (Flaxedil; $10 \mathrm{mg} / \mathrm{kg}$ supplemented every 45 min, Rhône-Poulenc Rorer) and artificially ventilated maintaining the expired $\mathrm{CO}_{2}$ near $4 \%$. Pools were constructed with the skin flaps surrounding the spinal cord and the hindlimb nerves and filled with warm mineral oil. Both body and pool temperature were kept near $38^{\circ} \mathrm{C}$ with radiant heat lamps. When necessary, a bilateral pneumothorax was performed to minimize respiratory movements and to stabilize intra-axonal recordings.

In the decerebrate cats, fictive locomotion, when not present spontaneously (Gossard, 1996), could be initiated by exteroceptive stimulation of various parts of the body or by electrical stimulation $(15-40 \mathrm{~Hz}$, $20-150 \mu \mathrm{A}$ ) with a low impedance tungsten electrode in the mesencephalic locomotor region (MLR) (Shik et al., 1966). In spinalized animals
(T13), episodes of fictive locomotion were induced by the injection of nialamide $(50 \mathrm{mg} / \mathrm{kg})$ and 3,4-dihydroxyphenylalanine (L-DOPA) methyl ester $(100-150 \mathrm{mg} / \mathrm{kg})$ (Jankowska et al., 1967a,b) or clonidine (250 $\mu \mathrm{g} / \mathrm{kg})$.

Recording and stimulation. A silver chloride ball electrode was placed on the cord dorsum to record the afferent volleys from the dorsal root entry zone. The threshold for the most excitable fibers in the nerve (1T) was determined as the stimulus strength required to just evoke a deflection in the cord dorsum potential. Stimulus intensity was expressed as a multiple of the threshold. Intra-axonal recordings were made with glass micropipettes filled with $\mathrm{K}^{+}$acetate $\left(10-25 \mathrm{M} \Omega\right.$ ) lowered at a $15^{\circ}$ angle from vertical, near the dorsal root entry zone of segments L6-L7 and up to $1.2 \mathrm{~mm}$ deep. Only axons with a $40 \mathrm{mV}$ spike, a membrane potential more negative than $-40 \mathrm{mV}$, and a stable DC signal were accepted for study.

Muscle afferents were identified by different physiological criteria (Gossard, 1996): (1) threshold for activation ( $<2.0 \mathrm{~T}$ for group I, 2.0 -5.0 T for group II); (2) the ability to follow electrical stimulation of the muscle nerve at high frequency $(>500 \mathrm{~Hz})$ with a short and constant latency and the absence of a prepotential on the evoked spike; and (3) conduction velocity as estimated by dividing the nerve length, measured at the end of the experiment, by the latency of the response. Unfortunately, group IA and IB fibers could not be fully characterized in such a preparation because of the limited access to muscles in the oil pool, cut nerves, and curarization. PADs were evoked by stimulating a peripheral muscle nerve (e.g., PBSt), usually with three shocks or pulses (p) at 300 $\mathrm{Hz}$ (Eccles et al., 1961) and with 2-5 T. This stimulation intensity usually evoked PADs of amplitude that could be easily measured without reaching the firing threshold.

Figure 1 is a schematic illustration of the neuronal circuitry involved in this study. Stimulation of peripheral muscle nerves, given at random times (up to three times per second) during the fictive locomotor cycle, was used to activate PAD interneurons (Fig. 1, right, PAD INs) mediating presynaptic inhibition via axoaxonic synapses at the terminals of the recorded muscle afferents. After successful PAD recording, the micropipette was drawn just outside the axon to record the extracellular field potential evoked by the same stimulation. As seen in Figure 1, left, the amplitude of the averaged extra-axonal potential was negligible. The inhibitory effect of PAD on synaptic transmission was evaluated by measuring the amplitude of the MSR with and without PAD. At first, heteronymous MSRs were evoked (see Fig. $7 A$ ) by stimulating the LGS nerve $(1 \mathrm{p}, 2 \mathrm{~T})$ while recording from the MG nerve or conversely. Then, the PBSt nerve was stimulated 20-25 msec before the MSR stimulation to evoke the MSR at the peak of PAD. In both situations, the MSR amplitude was measured from the rectified and low-pass filtered ENG activity. When possible, an intra-axonal recording of a stimulated group I fiber was performed simultaneously to directly measure the modulation of PAD amplitude during the same episode of, during a preceding, or a following episode of fictive locomotion.

ENG activities of the different muscle nerves were recorded using 


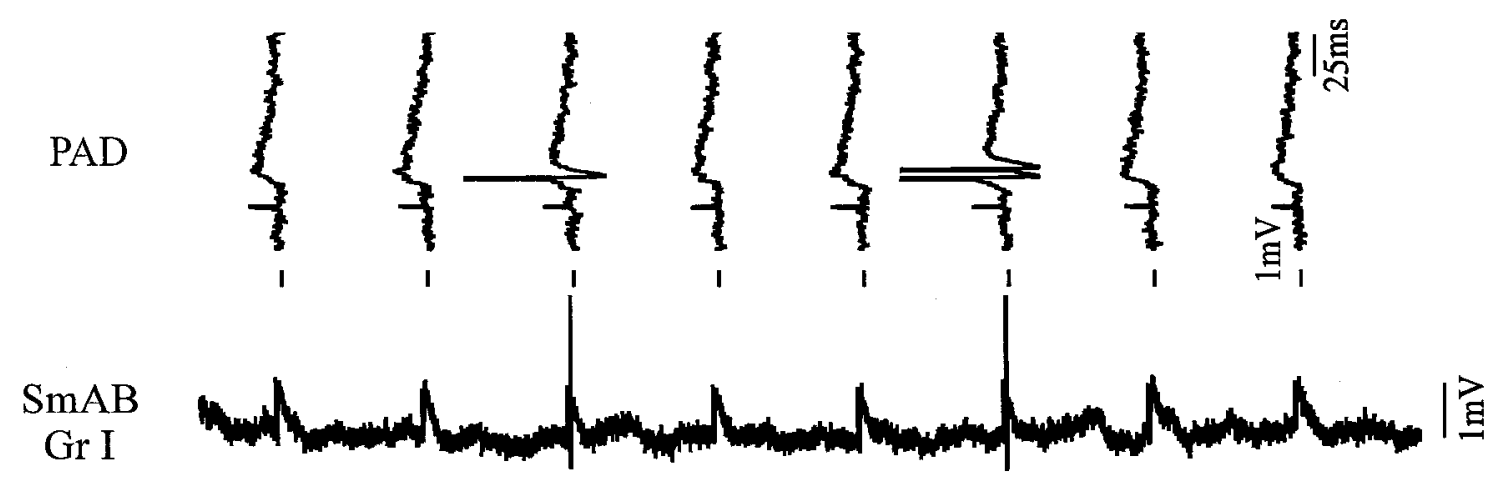

FDHL

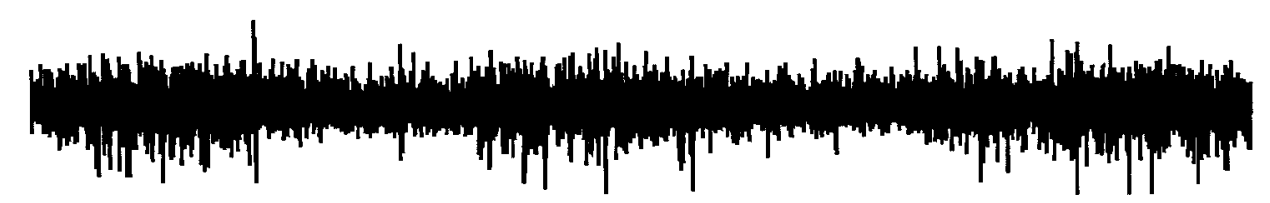

P1

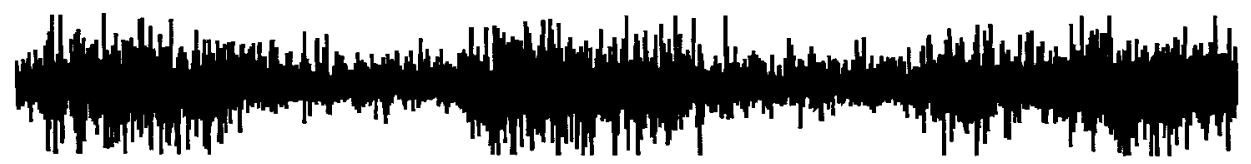

TA/EDL

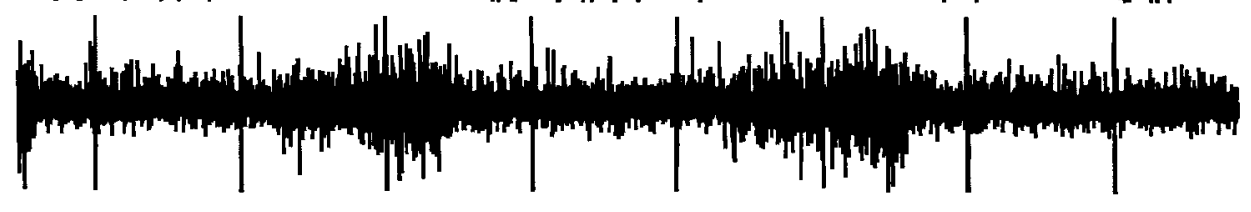

Stim PBST 3p 5T 300Hz

Figure 2. Intra-axonal recording of PADs during fictive locomotion. Traces, Top to bottom, Eight PADs (tilted $90^{\circ}$ ) recorded in a group I fiber from SmAB and evoked by PBSt stimulation $(3 \mathrm{p}, 5 \mathrm{~T}, 300 \mathrm{~Hz})$; continuous intra-axonal recording from the afferent; and locomotor ENG activities of FDHL, $\mathrm{Pl}$, and TA/EDL.

differential AC amplifiers. The signals were filtered $(10 \mathrm{~Hz}$ to $10 \mathrm{kHz})$ and visualized (Labview; National Instruments) to monitor the locomotor patterns. All signals were recorded on videotape after digitization (15 channels with $200 \mu \mathrm{sec}$ rise time/channel; Vetter 4000A).

Data collection and analysis. Tapes were played back off-line on an electrostatic printer (Gould ES-1000), and sections of data suited for analysis were digitized and analyzed with interactive custom-made software (Spinal Cord Research Center, University of Manitoba, Winnipeg, Canada).

The locomotor cycle, defined as the period between the onset of two successive bursts of ENG activity in flexors, was normalized to the duration of the averaged cycle and divided into 10 equal portions or "bins." All PADs occurring in a given bin were averaged over the entire locomotor episode. Phase plots of PAD amplitude were constructed by plotting the averaged integrated amplitude of PADs grouped in different bins as a percentage of the largest response (Gossard et al., 1990). These plots were aligned with the averaged rectified ENG activities of a flexor and an extensor, determining the locomotor phases, for examination of temporal relationships. The MSRs were also averaged within each bin of the fictive step cycle, and the averaged integrated amplitude of MSRs were used to construct phase plots of MSR amplitude with and without PAD (see Fig. 7).

Phase plots retained for statistical analysis had averages of at least 10 PADs in a majority of bins. At first, the Kolmogorov-Smirnov-Liliefors test was used to compare the shape and location of the sample distribution (standardized measures of PAD within a bin) to the standard normal distribution. This test confirmed that the sample variables did not follow normal distribution, and subsequent nonparametric tests were thus performed. The Mann-Whitney $U$ test was used to compare, pair by pair, the rank values of the variables from the bin with the largest mean with rank values of every other bin. This test was thus performed for each bin. The same statistical analysis was performed for phase plots of MSR amplitude with and without PAD. The "strength" of modulation was estimated by the number of significant bins. In Figure 7, SDs are illus- trated, and the significantly modulated bins are pointed out by asterisks $(p<0.05)$.

\section{RESULTS}

\section{Phasic modulation of PAD amplitude in} muscle afferents

The modulation of PAD amplitude was examined in detail and plotted (see Materials and Methods) for all selected trials. Examples of the raw recorded signals and the corresponding phase plot obtained from one axon are illustrated in Figures 2 and $3 A$, respectively. Figure 2 shows an intra-axonal recording of a SmAB group I fiber in which PADs were evoked during a period of fictive locomotion. Fictive step cycles, identified as alternating ENG activities in flexor (TA/EDL) and extensor (Pl) nerves, were induced by injection of nialamide and L-DOPA in a spinalized cat. The PADs in this axon were produced in response to PBSt nerve stimulation $(3 \mathrm{p}, 5 \mathrm{~T}, 300 \mathrm{~Hz})$ given in the various phases of the locomotor cycle and are also presented as traces tilted $90^{\circ}$ overlying the intra-axonal signal with a shorter time scale. In this case, the largest PADs (Fig. 2, PAD, third and sixth traces) occurred only during the flexor phase (TA/EDL bursts) and reached the firing threshold leading to antidromic discharges. The phasic modulation of PAD amplitude (without antidromic discharges) of this $\mathrm{SmAB}$ axon is also shown in the phase plot of Figure $3 A$. As previously depicted in Figure 2 by the presence of antidromic discharges, the averages confirmed that the maximal PAD amplitude occurred during the flexor phase. More specifically, the PAD amplitude in Figure $3 A$ was maximal at the 
A

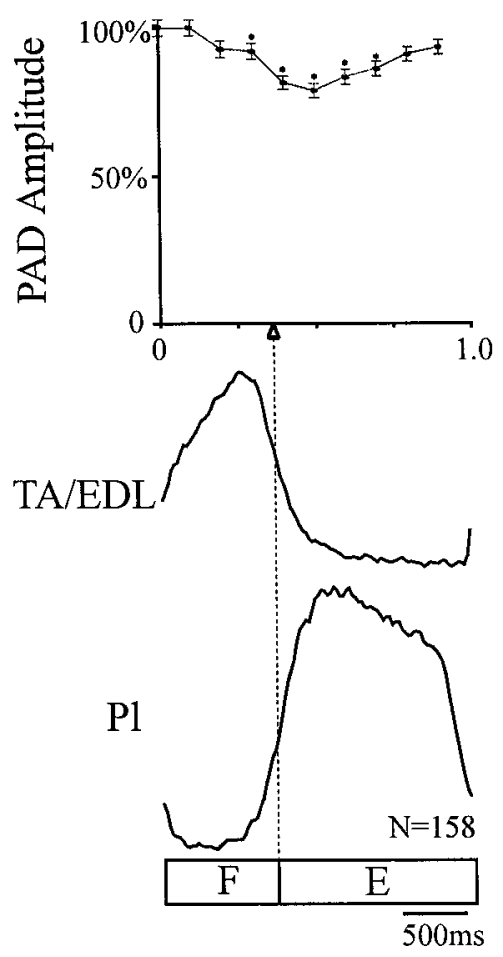

Stim PBSt 3p 5T 300Hz
B

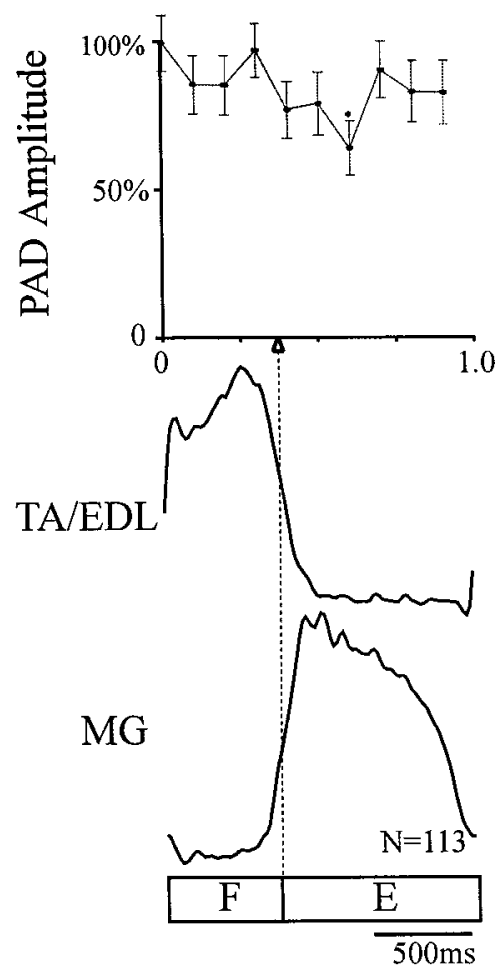

Stim PBSt 3p 5T 300Hz beginning of the flexor phase, decreased to reach a minimum in the first half of the extensor phase, and then increased again for the rest of that phase.

Overall, 70 trials were recorded in 51 fibers. Fifty-three trials, taken from 39 fibers (37 from group I and two from group II), were retained for analysis (see Materials and Methods). Thirtysix of 53 trials $(68 \%)$ from 30 muscle axons had at least one bin significantly different from the maximal one $(p<0.05)$ and were thus considered significantly modulated ( 7 of $11 \mathrm{Pl} ; 8$ of 12 LGS; 4 of $4 \mathrm{SmAB}$; 1 of $1 \mathrm{MG}$; 4 of $6 \mathrm{TA} / \mathrm{EDL} ; 6$ of $8 \mathrm{TiB}$; 6 of 10 FDHL; and 0 of 1 PBSt). The depth of modulation for the 36 trials varied from 13 to $80 \%$ ( $34.5 \%$ average). The results thus demonstrate that there was a significant phasic modulation of PAD amplitude in a majority of muscle afferents during fictive locomotion. Significant modulation was obtained in axons recorded from different locomotor preparations; 21 of 53 were taken from episodes of spontaneous locomotion in seven decerebrate cats, 9 of 53 from MLR-evoked locomotion in four decerebrate cats, 21 of 53 from eight spinal cats injected with nialamide and L-DOPA, and 2 of 53 patterns were recorded during fictive locomotion induced by the injection of clonidine in one spinal cat. Overall, the phasic modulation of PADs was observed in nine decerebrate cats ( 24 of 32 trials), as well as in eight spinal cats (12 of 21 trials), and no clear differences could be observed between the two.

The extent of modulation in PAD pathways present in both preparations (decerebrate vs spinal) could actually be compared within the same cat $(n=2)$. For example, Figure 3 depicts the PAD modulation obtained from two SmAB group I fibers recorded before $(B)$ and after $(A)$ spinalization. The PADs were evoked by the same PBSt nerve stimulation and were recorded during episodes of fictive stepping with similar characteristics (levels of ENG activities and cycle duration). The two patterns of modulation of PAD amplitude show some similarity, i.e., maximal PAD during the flexor phase and minimal during the early extension phase. However, although the depth of modulation appears larger in Figure $3 B$ than in Figure $3 A$, there was a smaller number of significantly modulated bins before spinalization, probably because there was more variability in the response amplitude (larger SDs). These results clearly indicate that the activity of spinal locomotor networks alone are able to phasically modulate the PAD pathways.

\section{Patterns of phasic modulation of PADs}

We further investigated whether the pattern of phasic modulation of PAD amplitude was the same for different types of afferents as was the case in cutaneous fibers (Gossard et al., 1990). For all trials, we compared the phases for maximal and minimal PAD amplitude, the depth of modulation, the pattern of modulation, i.e., the time course of amplitude modulation, and the number of bins significantly modulated. As illustrated in Figures 4-7, the results showed that there was clearly no single pattern in muscle afferents but that PAD modulation varied with every recorded afferent.

We first restricted our analysis to trials in which PADs were evoked by the same peripheral nerve stimulation, i.e., PBSt. Figure 4 shows examples of PAD modulation obtained in two muscle group I fibers coming from two different muscles of the ankle (TA/EDL and Tib), evoked in both cases by PBSt nerve stimulation during episodes of spontaneous fictive locomotion in the same decerebrate cat. Figure $4 A$ shows that the averaged PAD amplitude in the TA/EDL group I fiber was significantly modulated throughout the fictive locomotor cycle, with an impressive depth of modulation. The PAD amplitude was maximum 
A

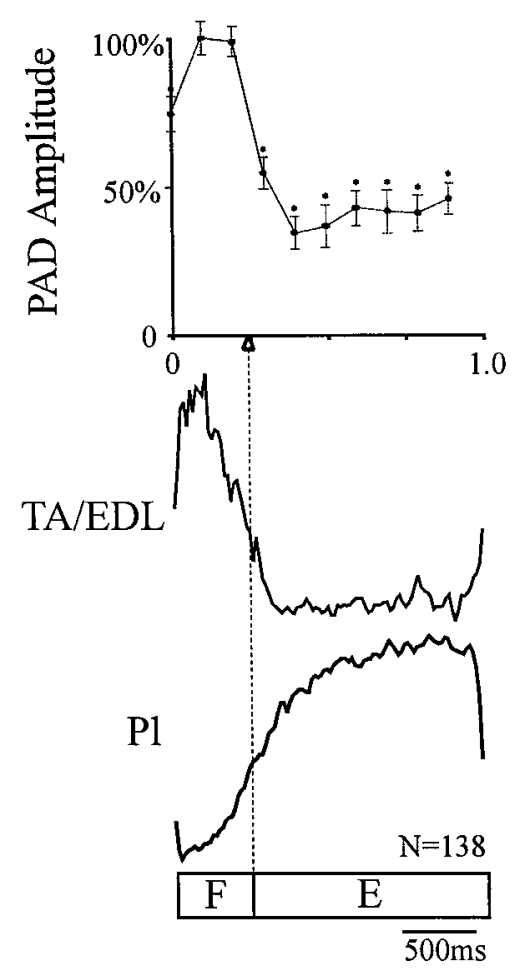

Stim PBSt 3p 5T 300Hz
B

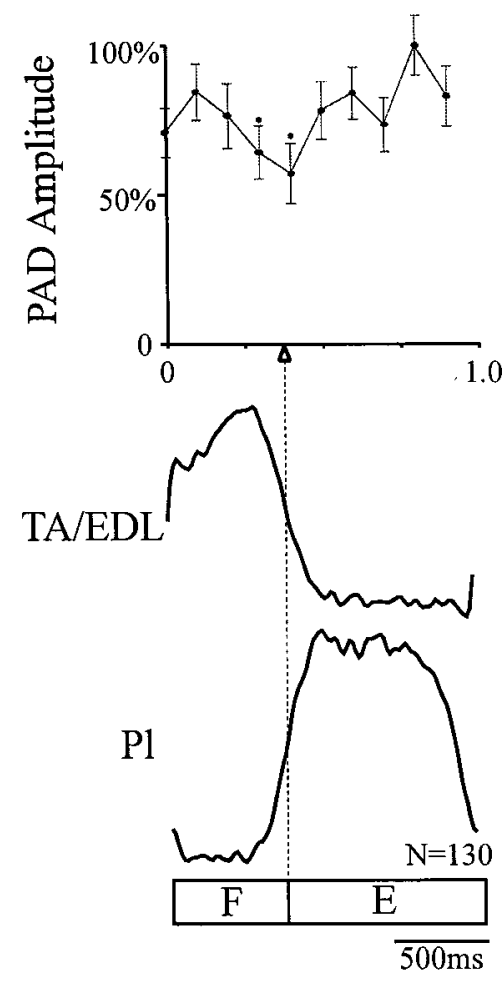

Stim PBSt 3p 5T 300Hz
Figure 4. Patterns of modulation of PAD amplitude in TA/EDL $(A)$ and Tib $(B)$ group I fibers recorded during spontaneous fictive locomotion in one decerebrate cat. Same outline as in Figure 3. during most of the flexor phase, decreased during the transition to the extensor phase, and became minimal throughout this latter phase. The averaged amplitude of PADs recorded in the Tib fiber, plotted in Figure $4 B$, was not as strongly modulated as in the TA/EDL fiber and followed a different pattern of phasic modulation. The PAD amplitude gradually decreased during the flexor phase to reach a minimum at the transition between the flexor and extensor phases; it then increased from this point to a maximum at the end of the extensor phase.

Differences in patterns of modulation in PAD amplitude were not only observed between afferent fibers coming from synergist or antagonist muscles but also between fibers coming from the same muscle. For example, we succeeded in recording one pair of fibers coming from the same muscle (two Pl, two FDHL, two Tib, and two LGS) in four different cats. In each pair, PADs were evoked by the stimulation of the same nerve during similar episodes of fictive locomotion. In every case, the comparison of patterns of modulation in both fibers revealed clear differences for the phases of maximum and minimum PAD amplitude and differences in the number of significant bins (Fig. 7B).

In Figure 5, we grouped all trials with a significant PAD modulation evoked by the stimulation of PBSt $(n=21)$. Figure 5 shows, for each trial, the maximal and minimal PAD amplitudes ( filled and open circles, respectively) relative to the fictive locomotor cycle, with the flexor and extensor phases separated by a short vertical line. The number of bins with significant modulation is also given for each trial. In Figure $5 A$, the trials are grouped according to muscles, and in Figure $5 B$, they are grouped according to the proportion of the flexor phase within the locomotor cycle, increasing from top to bottom. Figure $5 A$ shows that the maximal and minimal PAD amplitudes could occur in any of the 10 bins of the fictive step cycle, independent of the muscle of origin. Figure $5 B$ shows that the occurrence of maximal or minimal PAD amplitudes was not related to the locomotor pattern as monitored by the relative durations of flexor and extensor phases within the fictive step cycle.

The above results showed that, surprisingly, the precise PAD pattern varied with every recorded muscle afferent. We further investigated whether different afferent input could also evoke specific PAD patterns. We thus stimulated different muscle nerves and compared PAD patterns recorded in the same muscle afferent. Figure 6, $A$ and $B$, compares the patterns of modulation of PAD amplitude evoked by the stimulation of two different sources, a proximal muscle nerve (PBSt) and a distal muscle nerve (TA/EDL), recorded in the same Pl axon during fictive locomotion induced by nialamide and L-DOPA in a spinalized cat. Figure $6 A$ depicts the results obtained in response to PBSt nerve stimulation $(3 \mathrm{p}, 5 \mathrm{~T}, 300 \mathrm{~Hz})$ and shows that the amplitude of PADs was significantly modulated, with the maximum occurring at the beginning of the flexor phase and the minimum at the beginning of the extensor phase. In contrast (Fig. 6B), the PAD evoked by TA/EDL nerve stimulation at the same relative intensity $(3 \mathrm{p}, 5 \mathrm{~T}, 300 \mathrm{~Hz})$ was not significantly modulated. Various sources (two to four nerves) of PADs were investigated in seven axons. In every case, the number of bins significantly modulated, varied with every nerve, and only two axons showed similar phases for maximum and minimum PAD amplitude. However, we found that overall there was a larger proportion of trials, with a significant PAD modulation evoked by the stimulation of proximal muscle nerves (PBSt and SmAB; 25 of 33, 75.8\%) than among trials evoked by the stimulation of more distal muscles nerves (TA/EDL and GS; 11 of $19,57.9 \%$ ).

In summary, no clear tendencies in the patterns of modulation in PAD amplitude could be found among the 36 of 53 trials, even 

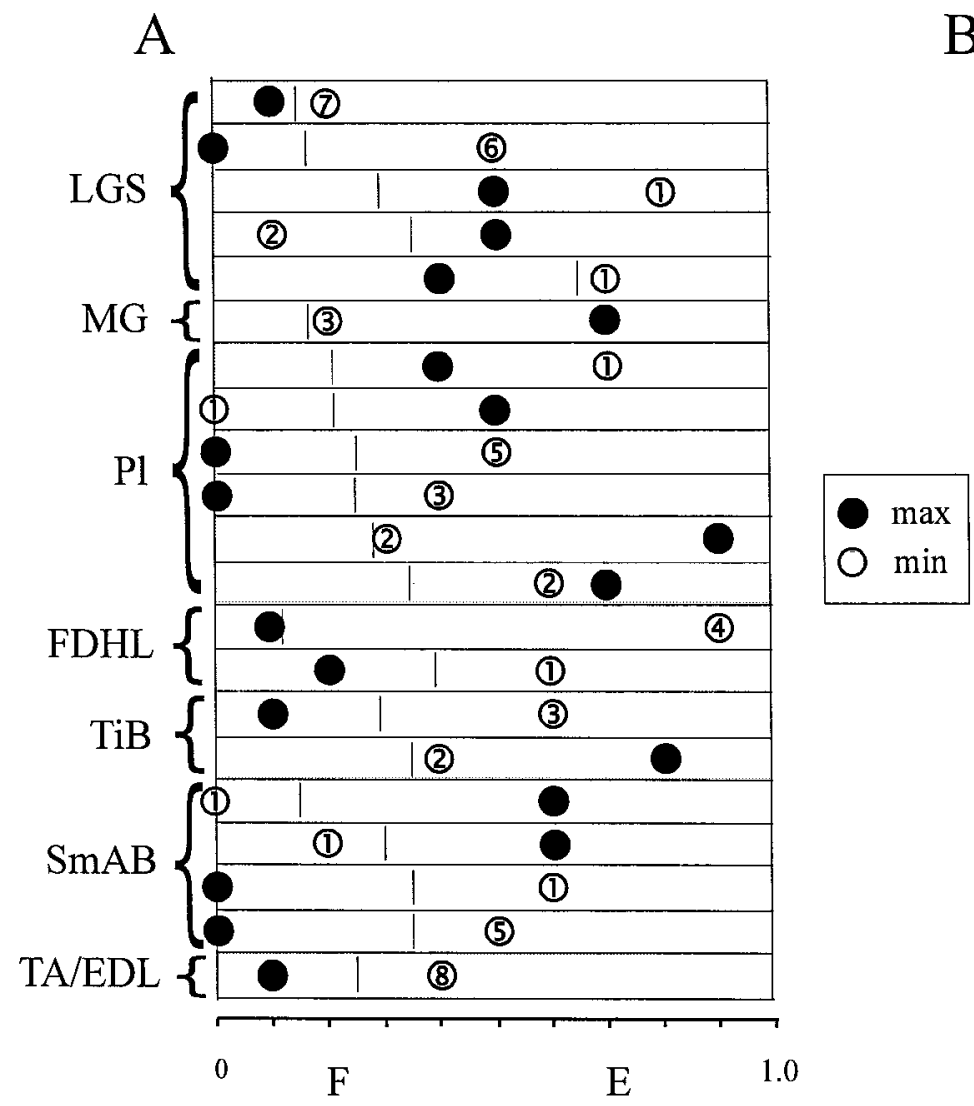

B

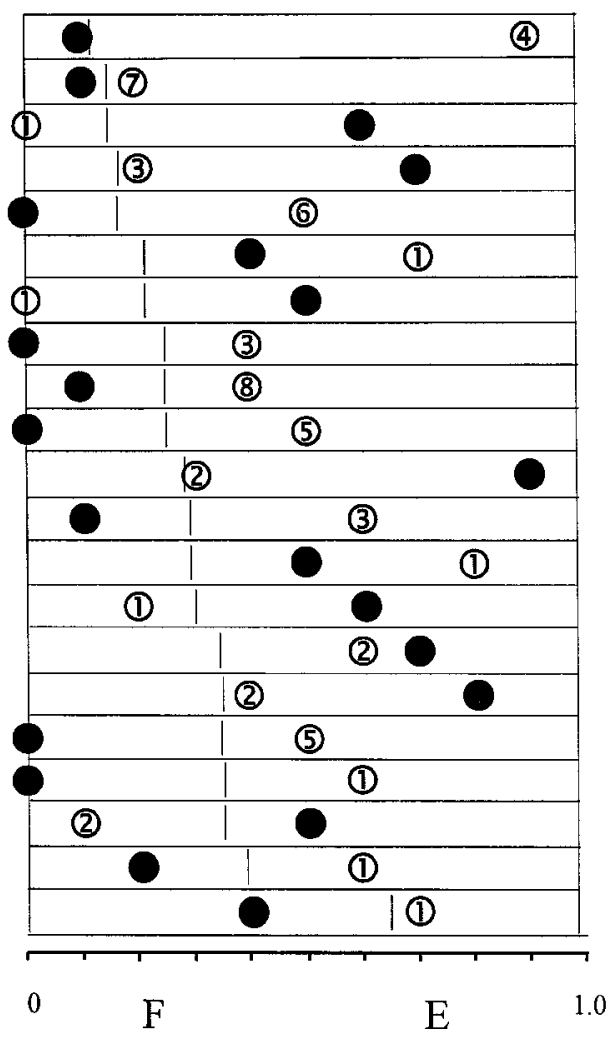

Figure 5. Phase plots of maximal and minimal PAD amplitude. PADs in all illustrated trials were evoked by stimulation of the same nerve (PBSt). A, Trials are grouped according to the muscle of origin as indicated on the left. Maximal and minimal amplitude of PADs are represented by filled and open circles, respectively. The numbers in the open circles are the number of bins significantly modulated. For each axon, the locomotor cycle is represented by a box, with the flexor $(F)$ and extensor $(E)$ phases separated by a short vertical line. $B$, Trials are grouped according to the proportion of the flexor phase within the fictive step cycle, increasing from top to bottom.

when we restricted our analysis to trials produced by the same stimulation or to afferent fibers coming from the same muscle. The results further suggest that the precise pattern of modulation depended on both the recorded afferent and the afferents producing the PAD.

\section{The effect of PAD on MSR during fictive locomotion}

Because PADs produce presynaptic inhibition, we assume that the observed phasic modulation of PAD amplitude in our experiments reflected phasic variations in presynaptic inhibition. The extent of the inhibitory action of PAD modulation during fictive locomotion in our preparations was studied by examining the MSR amplitude with and without PADs. Figure 7 illustrates a typical example of what was observed seven times in four cats. Figure $7 A$ is a schematic representation of the pathways involved in these tests. A heteronymous monosynaptic contact between an LGS fiber and a MG motoneuron is illustrated in Figure 7A, top, with the addition of an inhibitory synapse to the LGS axon terminal from PAD interneurons in the bottom. In both situations, an intra-axonal recording of the LGS fiber was performed while the MSR amplitude was measured from the MG ENG (see Materials and Methods). Examples of intra-axonal potentials and evoked MSRs (rectified and averaged) are given overlying the corresponding neurons in the schema. At first, LGS nerve was stimulated alone. As shown in Figure $7 A$, top, this stimulation evoked an action potential (truncated) in the LGS fiber and induced a heteronymous MSR in the MG nerve. Then, the LGS nerve stimulation was preceded by a PBSt nerve stimulation ( $3 \mathrm{p}$, $5 \mathrm{~T}, 300 \mathrm{~Hz}$ ) by $20-25 \mathrm{msec}$ to have the monosynaptic transmission on the top of the evoked PAD (note the action potential over the PAD in the LGS fiber). As shown in Figure $7 A$, bottom, the evoked MSR $(M S R+P A D)$ induced in this situation was clearly reduced, showing the inhibitory effect of PAD on the monosynaptic transmission.

Figure $7 B$ illustrates the patterns of modulation in MSR amplitude with and without PAD during a period of MLR-evoked fictive locomotion in a decerebrate cat. In Figure $7 B$, the unconditioned MSR (top phase plot, black line) showed a significant modulation, with a maximal amplitude during the extensor phase and a minimal amplitude during the flexor phase as described previously (Akazawa et al., 1982). In the same plot, one can see that the amplitude of the MSR preceded by PADs followed a similar pattern of modulation (gray line), with a maximum amplitude during the extension phase and a minimum during the flexor phase, but the depth of modulation was clearly reduced (overall reduction, $36 \%$ ). Note also the moderate decrease in SDs of the MSR amplitude after PADs. Similar results were seen in six other trials in which the MSR was reduced on average by $39 \%$. For the experiment illustrated in Figure $7 B$, PADs were recorded in two LGS group I fibers. The first one (LGS-1) was recorded simultaneously with the stimulation of MSRs, and the second LGS fiber (LGS-2) was recorded 28 min before the MSR stimulation during a similar episode of fictive stepping. The phase plots 


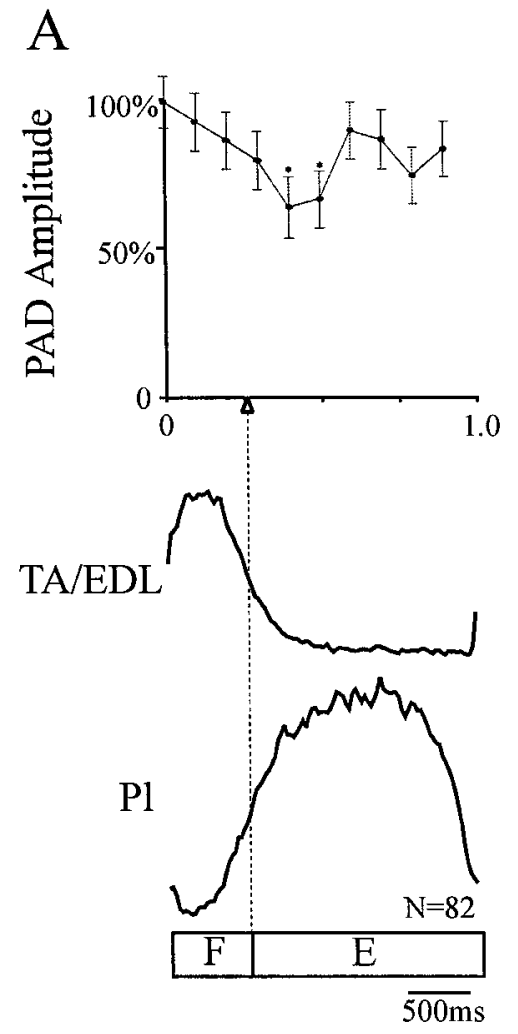

Stim PBSt 3p 5T 300Hz

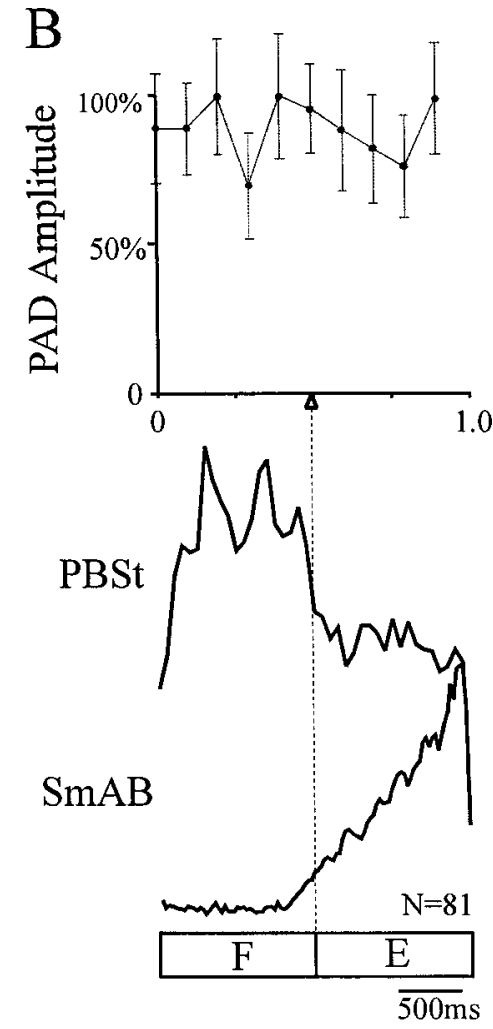

Stim TA/EDL 3p 5T 300Hz
Figure 6. Patterns of modulation of PAD amplitude produced by different sources of PAD in the same $\mathrm{Pl}$ group I fiber recorded during fictive locomotion induced by nialamide and L-DOPA in a spinal cat. Same outline as in Figure 3. for those two LGS fibers show quite different PAD modulation patterns. The PAD amplitude in LGS-1 was strongly modulated (seven bins), with a maximum during the flexion phase, whereas in LGS-2, the PAD amplitude was much less significantly modulated (one bin), with a maximum occurring during the second half of the extensor phase. There was no obvious relationship between the overall decrease in MSR amplitude (Fig. 7B, top phase plot, gray line) and the patterns of PAD modulation in LGS-1 or LGS-2. In other words, the PAD patterns of neither axon can explain the phasic modulation of MSR amplitude. The two superimposed traces in Figure $7 B$, bottom, are the ENG activities in the MG nerve in both conditions (with and without PAD) and show that there were no important differences in locomotor activities in the MG nerve during these two tests, suggesting that MSR reduction did not result from major changes of excitability in MG motoneurons but was secondary to the PADs.

\section{DISCUSSION}

\section{CPG-dependent modulation of transmission in PAD pathways}

Intra-axonal recordings of identified muscle afferents have revealed that PAD amplitude, evoked by muscle nerve stimulation, was phasically modulated during fictive locomotion in a majority of muscle afferents (30 of 39, 77\%). However, not all stimulated nerves led to significantly modulated PADs in a given axon (36 of 53 trials, 68\%). Also, PAD amplitude was significantly modulated during fictive locomotion in both decerebrate and spinal cats and indicates that the spinal cord networks alone are able to modulate the transmission in PAD pathways. It is thus suggested that the CPG for locomotion is primarily responsible for the phasic modulation of PADs in muscle group I fibers (Duenas et al., 1990;
Gossard and Rossignol, 1990; Gossard et al., 1990; El Manira et al., 1991, 1997; Cattaert et al., 1992; Nusbaum et al., 1997). The potential contribution of supraspinal structures to locomotor presynaptic mechanisms remains unknown presently.

Several hypotheses can be proposed to explain how PAD amplitude is modulated in a phase-dependent manner by spinal locomotor networks. One possibility is the spontaneous cyclic changes of polarization of primary afferents accompanying locomotion (Baev and Kostyuk, 1982; Dubuc et al., 1988; Duenas and Rudomin, 1988; Gossard et al., 1989, 1991). Such CPG-related waves of polarization could alter the excitability of the stimulated, as well as recorded, afferent fibers. However, a previous study showed that the CPG-related waves of polarization followed a similar pattern in all muscle group I afferents (Gossard et al., 1991). This lack of specificity cannot explain the wide variety of PAD patterns observed in this study. Moreover, a recent study from our laboratory suggests that the CPG-related waves of polarization in group IA afferents have little effect on the monosynaptic transmission to motoneurons (Gossard, 1996). A simpler explanation is that the CPG affects the transmission in the PAD pathways by changing the excitability of the intercalated interneurons (Fig. 1 $A$, arrow).

\section{Organization of presynaptic inhibitory pathways}

A simple pattern of modulation of PAD was reported previously for all cutaneous fibers, with maximum amplitude during the extensor phase of the fictive step cycle, regardless of whether they innervated the dorsal or ventral surfaces of the hindpaw (Gossard et al., 1990). In contrast, no clear or simple tendencies in PAD patterns were detected in our sample of muscle afferents, regardless of whether we focused on patterns produced by the same 

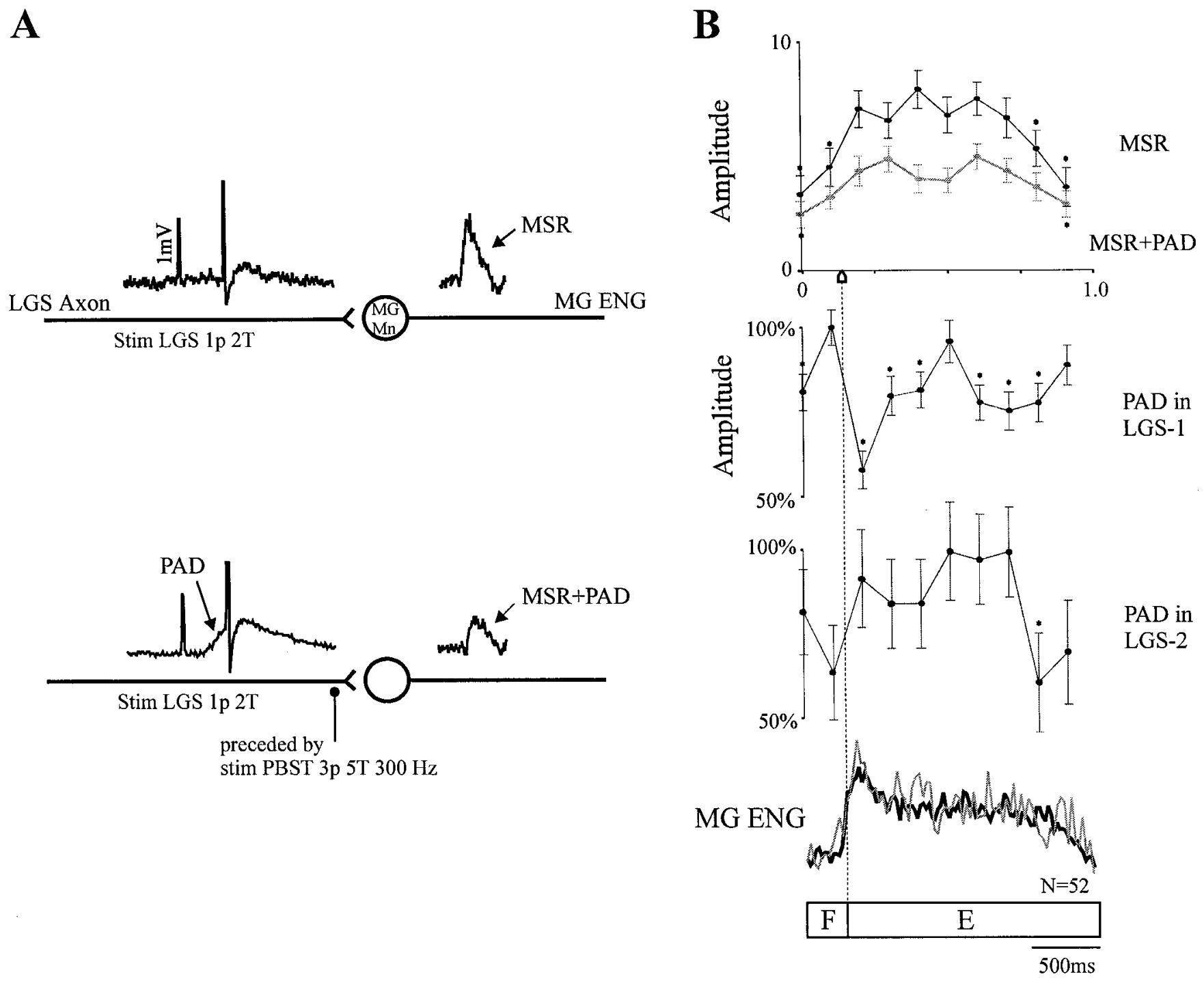

Figure 7. Effect of PAD on MSR amplitude during fictive locomotion. A, Schematic representation of the pathways involved. Top to bottom, Stimulation of the LGS nerve ( $1 \mathrm{p}, 2 \mathrm{~T}$ ) evoked an action potential in the LGS fiber contacting the MG motoneuron $(M G M n)$ and induced a heteronymous MSR measured from the rectified MG ENG; and stimulation of the LGS nerve (1 p, $2 \mathrm{~T})$ was then preceded by the stimulation of PBSt (3 p, $5 \mathrm{~T}, 300 \mathrm{~Hz})$, which evoked an action potential on the top of a PAD in the LGS fiber and induced a reduced MSR. $B$, Top to bottom, Phase plot of the averaged amplitude of MSRs obtained with ( gray) and without (black) PAD; phase plots of the averaged amplitude of PADs recorded in two LGS group I fibers; and superimposed rectified MG locomotor ENG activities recorded with (gray) and without (black) PAD.

stimulation (Fig. $5 A$ ) or on fibers originating from the same muscle (Fig. $7 B$ ). Also, it is improbable that the different PAD patterns were strictly related to the different patterns of fictive locomotion (Fig. 5B). Overall, the results showed that the precise pattern of modulation depended on both the recorded fiber and the fibers producing the PAD (Fig. 6).

The wide variety in patterns of modulation of PAD amplitude may thus indicate that the CPG influence is exerted via multiple subsets of PAD interneurons with a complex connectivity from and to muscle group I afferents. First, because the stimulation of two different peripheral nerves evokes two different PAD modulation patterns in the same fiber, a group I fiber should be contacted by at least two different subsets of PAD interneurons. Second, because two afferents from the same muscle may display different PAD modulation patterns evoked by the same stimulation, each group I fiber should be contacted also by specific subsets of PAD interneurons. Complex connectivity of PAD interneurons have already been proposed to explain differences in intraspinal thresholds between group IA and IB fibers and also between branches of the same fiber in anesthetized cats $(\mathrm{Ru}-$ domin et al., 1987, 1998; Rudomin, 1990; Equibar et al., 1994, 1997; Enriquez et al., 1996; Quevedo et al., 1997). Surprisingly, a similar reduction in intraspinal threshold was found during the flexor phase of the fictive step cycle for both group IA and IB fibers and for afferent terminals in the intermediate nucleus and motor nucleus (Duenas and Rudomin, 1988). On the other hand, the diversity of PAD patterns observed in this study using intraaxonal recordings did not follow a simple pattern and supports strongly that PAD interneurons are indeed functionally subdivided by the CPG for locomotion. Also, a complete characterization of group IA and IB afferents in our sample could have helped us classify PAD patterns. However, the observed PAD patterns 
did not fall into two categories, as predicted by only two separate PAD subpopulations.

\section{Presynaptic inhibition and the MSR pathway}

The MSR pathway may represent a particular case among spinal cord pathways, because each motoneuron in a motor pool receive synaptic contacts from almost all group IA afferents from the homonymous muscle (Mendell and Henneman, 1971). Our results showed that there was an overall reduction of the MSR amplitude (39\% average) by PADs during fictive locomotion but that there was no clear relationship between the phase of maximum PAD amplitude in any single group I fiber and the phase of maximum MSR amplitude (Fig. $7 B$ ). This lack of relationship is probably attributable to the fact that the MSR in this study was evoked by an electrical stimulation of a peripheral nerve, which activates synchronously all group I fibers. In a previous study from this laboratory, the monosynaptic EPSPs evoked by a single group IA afferent (unitary EPSPs) were recorded in the target motoneuron during fictive locomotion and showed a clear phasedependent modulation (Gossard, 1996). However, in the case in which all group I fibers are stimulated synchronously (composite EPSPs), one would not expect a clear phase-dependent modulation of EPSPs because of the mix of all the different PAD patterns in the group I fibers. Indeed, monosynaptic EPSPs recorded in motoneurons in studies using electrical nerve stimulation showed a weak phase-dependent modulation but a clear "tonic" inhibition (Schomburg and Behrends, 1978; Shefchyk et al., 1984; Gosgnach et al., 1998). This is also consistent with the reduced "gain" of the MSR pathway observed in humans from standing to walking, which was attributed to presynaptic inhibition (Stein and Capaday, 1988). As discussed in previous studies (Jordan, 1983; Shefchyk et al., 1984; Burke, 1987; Burke et al., 1989), postsynaptic mechanisms, such as the locomotor drive potential, seem to be responsible for most of the phase-dependent modulation of the MSR. In brief, because of its particular massive connectivity, the MSR pathway may not take full advantage of specific PAD patterns.

Another possible role for the widely distributed PAD patterns in group I afferents could be to decrease the variability of MSR (Fig. 7B). Indeed, Rudomin and Dutton (1969a,b) provided evidence that presynaptic modulation of group IA synaptic transmission was an important source of excitability fluctuation during the MSR (Gossard et al., 1994b). Conditioning inputs from peripheral nerves, producing a wide variety of PAD patterns as observed here, could decrease considerably the MSR variability by reducing the correlation of excitability fluctuations between motoneurons (Rudomin and Madrid, 1972).

\section{Functional considerations}

The possible role of presynaptic inhibition in the sensory control of motor tasks has been discussed previously in several species (Clarac et al., 1992; Wolf and Burrows, 1995; Nusbaum et al., 1997; El Manira et al., 1998; Rudomin et al., 1998). As for locomotion in mammals, it is difficult to infer what PAD patterns would result from the combined sensory feedback of cutaneous, joint, muscle, and other afferents activated by the stepping movements. Our results suggest that different stimulated afferents would evoke different PAD patterns (Fig. 6). It is almost technically impossible to record PADs during real walking, but previous studies succeeded in recording antidromic discharges in dorsal rootlets during treadmill locomotion of decerebrate cats (Beloozerova and Rossignol, 1994, 1995). It was reported that bursts of antidromic discharges could start or end in different phases of the step cycle, suggesting that the underlying PADs may reach maximal amplitude at different moments of the step cycle, as described in this study.

Finally, the complex organization of PAD interneurons as proposed above may also allow very precise and subtle control of synaptic transmission in highly focalized regions of the spinal cord. As suggested previously (Equibar et al., 1994, 1997; Quevedo et al., 1997; Rudomin et al., 1998), specific branches of the same muscle afferent could be controlled independently, depending on the task. For example, during a voluntary extension of the ankle in humans, quadricep afferent terminals show different levels of presynaptic inhibition, depending on whether they end on motoneurons recruited for the task or not (Hultborn et al., 1987). One could easily extend this view to include spinal interneurons. For example, the terminals from one afferent ending on spinal interneurons of a segmental pathway and the terminals from the same afferent ending on a ascending pathway could be differentially controlled by specific subsets of PAD interneurons. We may thus further propose that the connectivity of PAD interneurons in the spinal cord could be organized, not according to the type of primary afferents, but rather, according to their postsynaptic targets.

\section{REFERENCES}

Akazawa K, Aldridge JW, Steeves JD, Stein RB (1982) Modulation of stretch reflexes during locomotion in the mesencephalic cat. J Physiol (Lond) 329:553-567.

Baev KV, Kostyuk PG (1982) Polarization of primary afferent terminals of lumbosacral cord elicited by the activity of spinal locomotor generator. Neuroscience 7:1401-1409.

Beloozerova IN, Rossignol S (1994) Antidromic activity of dorsal root filaments during treadmill locomotion in thalamic cats. Soc Neurosci Abstr 20:1755.

Beloozerova IN, Rossignol S (1995) Antidromic activity in uncut dorsal roots of the cat. Soc Neurosci Abstr 21:413.

Bévengut M, Clarac F, Cattaert D (1997) Antidromic modulation of a proprioceptor sensory discharge in crayfish. J Neurophysiol 78:1180-1183.

Burke RE (1987) Synaptic efficacy and the control of neuronal inputoutput relations. Trends Neurosci 10:42-45.

Burke RE, Fleshman JW, Segev I (1989) Factors that control the efficacy of group Ia synapses in alpha-motoneurons. J Physiol (Paris) 83:133-140.

Cattaert D, El Manira A, Clarac F (1992) Direct evidence for presynaptic inhibitory mechanisms in crayfish sensory afferents. J Neurophysiol 67:610-624.

Clarac F, El Manira A, Cattaert D (1992) Presynaptic control as a mechanism of sensory-motor integration. Curr Opin Neurobiol 2:764-769.

Conway BA, Hultborn H, Kiehn O (1987) Proprioceptive input resets central locomotor rhythm in the spinal cat. Exp Brain Res 68:643-656.

Dubuc R, Cabelguen J-M, Rossignol S (1988) Rhythmic fluctuations of dorsal root potentials and antidromic discharges of primary afferents during fictive locomotion in the cat. J Neurophysiol 60:2014-2036.

Duenas SH, Rudomin P (1988) Excitability changes of ankle extensor group Ia and Ib fibers during fictive locomotion in the cat. Exp Brain Res 70:15-25.

Duenas SH, Loeb GE, Marks WB (1990) Monosynaptic and dorsal root reflexes during locomotion in normal and thalamic cats. J Neurophysiol 63:1467-1476.

Eccles JC, Eccles RM, Magni F (1961) Central inhibitory actions attributable to presynaptic depolarization produced by muscle afferent volleys. J Physiol (Lond) 159:147-166.

El Manira A, DiCaprio RA, Cattaert D, Clarac F (1991) Monosynaptic interjoint reflexes and their central modulation during fictive locomotion in crayfish. Eur J Neurosci 3:1219-1231.

El Manira A, Tegnér J, Grillner S (1997) Locomotor-related presynaptic modulation of primary afferents in the lamprey. Eur $\mathrm{J}$ Neurosci 9:696-705. 
El Manira A, Parker D, Krieger P, Wikström M, Grillner S (1998) Presynaptic inhibition of synaptic transmission from sensory, interneuronal, and supraspinal neurons to spinal target cells in lamprey. In: Presynaptic inhibition and neural control (Rudomin P, Romo R, Mendell LM, eds), pp 329-348. New York: Oxford UP.

Enriquez M, Jiménez I, Rudomin P (1996) Segmental and supraspinal control of synaptic effectiveness of functionally identified muscle afferents in the cat. Exp Brain Res 107:391-404.

Equibar JR, Quevedo J, Jiménez I, Rudomin P (1994) Selective cortical control of information flow through different intraspinal collaterals of the same muscle afferent fiber. Brain Res 643:328-333.

Equibar JR, Quevedo J, Jiménez I, Rudomin P (1997) Selective cortical and segmental control of primary afferent depolarization of single muscle afferents in the cat spinal cord. Exp Brain Res 113:411-430.

Gosgnach S, Quevedo J, Fedirchuk B, McCrea D (1998) Tonic presynaptic reduction of monosynaptic IA EPSPs during fictive locomotion. Proc NY Acad Sci, in press.

Gossard J-P (1996) The control of transmission in muscle group IA afferents during fictive locomotion in the cat. J Neurophysiol 76:4104-4112.

Gossard J-P, Rossignol S (1990) Phase-dependent modulation of dorsal root potentials evoked by peripheral nerve stimulation during fictive locomotion in the cat. Brain Res 537:1-13.

Gossard J-P, Cabelguen J-M, Rossignol S (1989) Intra-axonal recordings of cutaneous primary afferents during fictive locomotion in the cat. J Neurophysiol 62:1177-1187.

Gossard J-P, Cabelguen J-M, Rossignol S (1990) Phase-dependent modulation of primary afferent depolarization in single cutaneous primary afferents evoked by peripheral stimulation during fictive locomotion in the cat. Brain Res 537:14-23.

Gossard J-P, Cabelguen J-M, Rossignol S (1991) An intracellular study of muscle primary afferents during fictive locomotion in the cat. J Neurophysiol 65:914-926.

Gossard J-P, Brownstone RM, Barajon I, Hultborn H (1994a) Transmission in a locomotor-related group Ib pathway from hindlimb extensor muscles in the cat. Exp Brain Res 98:213-228.

Gossard J-P, Floeter M-K, Chang T, Kawai Y, Schiff SJ, Burke RE (1994b) Fluctuations of excitability in the monosynaptic reflex pathway to lumbar motoneurons in the cat. J Neurophysiol 72:1227-1239.

Grillner S (1981) Control of locomotion in bipeds, tetrapods and fish. In: Handbook of physiology, The nervous system II (Brookhart JM, Mountcastle VB, eds), pp 1179-1236. Bethesda, MD: American Physiological Society.

Hiebert GW, Whelan PJ, Prochazka A, Pearson KG (1996) Contribution of hindlimb flexor muscle afferents to the timing of phase transitions in the cat step cycle. J Neurophysiol 75:1126-1137.

Hultborn H, Meunier S, Pierrot-Deseilligny E, Shindo M (1987) Changes in presynaptic inhibition of Ia fibres at the onset of voluntary contraction in man. J Physiol (Lond) 389:757-772.

Ito F (1968) Recovery curves of thresholds of muscle spindle, leaf-like and tendon receptors in the frog sartorius muscle after an antidromic discharge. Jpn J Physiol 18:731-745.

Lindblom UF (1958) Excitability and functional organization within a peripheral tactile unit. Acta Physiol Scand Suppl 153:1-84.

Loewenstein WR (1959) The generation of electric activity in a nerve ending. Ann NY Acad Sci 81:367-387.

Jankowska E, Jukes MGM, Lund S, Lundberg A (1967a) The effect of DOPA on the spinal cord. V. Reciprocal organization of pathways transmitting excitatory action to alpha motoneurones of flexors and extensors. Acta Physiol Scand 70:369-388.

Jankowska E, Jukes MGM, Lund S, Lundberg A (1967b) The effect of DOPA on the spinal cord. VI. Half-centre organization of interneurones transmitting effects from the flexors reflex afferents. Acta Physiol Scand 70:389-403.

Jordan LM (1983) Factors determining motoneuron rhythmicity during fictive locomotion. Symp Soc Exp Biol 37:423-444.

McCrea DA, Shefchyk SJ, Stephens MJ, Pearson KG (1995) Disynaptic group I excitation of synergist ankle extensor motoneurones during fictive locomotion in the cat. J Physiol (Lond) 487:527-539.

Ménard A, Leblond H, Gossard J-P (1997) Modulation of primary afferent depolarization in muscle group I fibres during fictive locomotion in the cat. Soc Neurosci Abstr 23:761.

Mendell LM, Henneman E (1971) Terminals of Ia fibers: Location, density and distribution within a pool of 300 homonymous motoneurons. J Neurophysiol 34:171-187.

Nicoll RA, Alger BE (1979) Presynaptic inhibition: transmitter and ionic mechanisms. Int Rev Neurobiol 21:217-258.

Nusbaum MP, El Manira A, Gossard J-P, Rossignol S (1997) Presynaptic mechanisms during rhythmic activity in vertebrates and invertebrates. In: Neurons, networks, and motor behavior (Stein PSG, Grillner S, Selverston AI, Stuart DG, eds), pp 237-253. Cambridge, MA: MIT.

Pearson KG, Duysens J (1976) Function of segmental reflexes in the control of stepping in cockroaches and cats. In: Neural control of locomotion (Herman RM, Grillner S, Stein S, Stuart DC, eds), pp 529-537. New York: Plenum.

Quevedo J, Eguibar JR, Lomeli J, Rudomin P (1997) Patterns of connectivity of spinal interneurons with single muscle afferents. Exp Brain Res 115:387-402.

Rossignol S (1996) Neural control of stereotypic limb movements. In: Handbook of physiology. Exercise: Regulation and integration of multiple systems, Sect 12 (Rowell CB, Sheperd JJ, eds), pp 173-216. Bethesda, MD: American Physiology Society.

Rudomin P (1990) Presynaptic inhibition of muscle spindle and tendon organ afferents in the mammalian spinal cord. Trends Neurosci 13:499-505.

Rudomin P, Dutton H (1969a) Effects of conditioning afferent volleys on variability of monosynaptic responses of extensor motoneurons. J Neurophysiol 32:140-157.

Rudomin P, Dutton H (1969b) Effects of muscle and cutaneous afferent nerve volleys on excitability fluctuations on Ia terminals. J Neurophysiol $32: 158-169$.

Rudomin P, Madrid J (1972) Changes in correlation between monosynaptic responses of single motoneurons and in information transmission produced by conditioning volleys to cutaneous nerves. J Neurophysiol 35:44-64.

Rudomin P, Solodkin M, Jiminez I (1987) Synaptic potentials of primary afferent fibers and motoneurons evoked by single intermediate nucleus interneurons in the cat spinal cord. J Neurophysiol 57:1288-1313.

Rudomin P, Jiménez I, Quevedo J (1998) Selectivity of the presynaptic control of synaptic effectiveness of group I afferents in the mammalian spinal cord. In: Presynaptic inhibition and neural control (Rudomin P, Romo R, Mendell LM, eds), pp 282-302. New York: Oxford UP.

Schmidt RF (1971) Presynaptic inhibition in the vertebrate central nervous system. Ergebn Physiol 63:20-101.

Schomburg ED, Behrends HB (1978) The possibility of phasedependent monosynaptic and polysynaptic Ia excitation to homonymous motoneurones during fictive locomotion. Brain Res 143:533-537.

Shefchyk SJ, Stein RB, Jordan LM (1984) Synaptic transmission from muscle afferents during fictive locomotion in the mesencephalic cat. J Neurophysiol 51:986-997.

Shik ML, Severin FV, Orlovsky GN (1966) Control of walking and running by means of electrical stimulation of mid-brain. Biofizika 11:659-666.

Stein RB, Capaday C (1988) The modulation of human reflexes during functional motor tasks. Trends Neurosci 11:328-332.

Toennies JF (1939) Conditioning of afferent impulses by reflex discharges over the dorsal roots. J Neurophysiol 2:515-525.

Wolf H, Burrows M (1995) Proprioceptive sensory neurons of a locust leg receive rhythmic presynaptic inhibition during walking. J Neurosci 15:5623-5636.

Yang JF, Whelan PJ (1993) Neural mechanisms that contribute to cyclical modulation of the soleus H-reflex in walking in humans. Exp Brain Res 95:547-556. 